\title{
Principles and Practice of Clinical Research
}

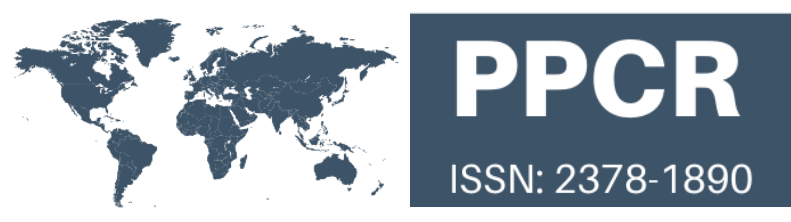

\section{Trimodal treatment for localized high-risk prostate cancer: A design of a phase II, multi-center, open- label, randomized, parallel-group study to compare the effectiveness and safety of trimodal treatment for patients with localized high-risk prostate cancer.}

\author{
R. Paz-Manrique ${ }^{1 *}$, LI. Ter ${ }^{2}$, I. Alnadhari ${ }^{3}$, S. Qayyum ${ }^{4}$, N. Abu Hussein ${ }^{5}$, B. Vieira Carneiro ${ }^{6}$, C. Sakuma \\ de Oliveira ${ }^{7}$, BE. Osman Elhag ${ }^{8}$, M. Martines da Costa ${ }^{9}$, R. Santos Bittencourt Silva ${ }^{10}$, RJ. Montenegro \\ Carrera $^{11}$, M. Antunes Kasa ${ }^{12}$, AA. Toribio Mejia ${ }^{13}$, R. Silva de Paula Rocha ${ }^{14}$, M. Elamin Faris ${ }^{15}$, AJ. \\ Barragan Berlanga ${ }^{16}$, L. Furtado Campos ${ }^{17}$, HH. Elamin ${ }^{18}$, F. González Mc.Cawley ${ }^{19}$, S. Szylewicz ${ }^{20}$, S. \\ Hijazi Vega ${ }^{21}$, A. Espinoza Briones ${ }^{22}$, G. Bartié Rossi ${ }^{23}$, C. Pires Camargo ${ }^{24}$ \\ *Corresponding authors: Roberto Paz-Manrique MD, Dirección Científica y Académica, Oncosalud AUNA, Lima, Perú, \\ robertopazm@hotmail.com
}

Rest of author's affiliation at the end of the manuscript.

Received December 16, 2019; accepted May 3, 2020; published May 11, 2020.

\begin{abstract}
:
Background: Prostate cancer is the second most prevalent neoplasm in men worldwide and the first leading cause of death around the globe. High-risk prostate cancer represents 15\% of all prostate cancer patients and, by definition, multimodal treatments are encouraged in order to achieve control of the disease, and even potential cure. Available treatments for such patients have been compared and there is no definitive evidence related to the superiority between surgery (radical prostatectomy - RP) and radiation therapy with or without hormone therapy.

Aims: To compare two multimodal treatment of high-risk prostate cancer: radical prostatectomy associate to adjuvant external radiation therapy and androgen deprivation therapy versus external radiation therapy plus androgen deprivation therapy. Main outcome: time to PSA recurrence. Secondary outcomes: metastasis free survival, defined as time to first clinical or radiological progression or death from any cause; overall survival, adverse events according to graduation of Common Terminology Criteria for Adverse Events (CTCAE) v5.0 and, quality of life assessed by Expanded Prostate Cancer Index Composite (EPIC) questionnaire life.

Methods: This is a proposed design and protocol for a phase II, prospective, randomized, open-label, and multicenter trial, including a total of 322 patients with localized high-risk prostate cancer. Outcomes were already described in the Aims section. 5 tertiary hospitals will be selected in Brazil, the intervention proposed is the trimodal treatment and the control group will receive standard of care, which consists in external radiation therapy plus hormonal therapy. Patients will have follow-up for 5 years.

Potential impact of the study: At the best of our knowledge, there aren't any randomized controlled trials to analyze the trimodal treatment. The possible outcomes of this study will offer a better evidence to high-risk prostate cancer and its results may contribute to change the standard treatment in this set of patients by reducing significantly local recurrence and offering a potential cure for this malady.
\end{abstract}

Keywords: Prostatic neoplasm, Combined Modality Therapy, prostatectomy, radiotherapy, Androgen Antagonists 


\section{INTRODUCTION}

Prostate cancer is the second most common cancer in men worldwide. According to World Health Organization (WHO) the incidence of prostate cancer is over 1.2 million cases and 358,000 deaths annually (Siegel, Miller, \& Jemal, 2019). The definition of high-risk localized prostate cancer is those with serum PSA $>20 \mathrm{ng} / \mathrm{mL}$ or Gleason score 8-10 or clinical stage T2c or higher (Mottet et al., 2017). It accounts for approximately $15 \%$ of all prostate cancer cases (Chang, Autio, Roach, \& Scher, 2014).

Standard of care treatments include two bimodal regimens: radical prostatectomy (RP), which consists on the removal of the prostate gland and seminal vesicles, associated with adjuvant external beam radiotherapy (EBRT - by using a device that emits radiation, solid tumors can be targeted in order to reduce or disappear malignancy) and EBRT associated with long term androgen deprivation therapy (ADT - Prostate cancer is considered to be fueled by testosterone. By cutting the supply of testosterone, prostate cancer can be controlled). It's been described that, for this subset of patients, EAU guidelines (Mottet et al., 2017) recommend multimodal treatments in order to decrease local recurrence, which will be very likely for these subjects.

RP plus EBRT vs RP alone resulted in improvement of median metastasis-free survival of, respectively, 14.7 years vs 12.9 years, HR 0.71 (95\% CI 0.54- 0.94; $\mathrm{p}=0.016$ ) (Thompson et al., 2009), and longer biochemical progression-free survival, HR 0.49 (95\% CI 0.41-0.59; $\mathrm{p}<0.0001$ ) (Bolla et al., 2012).

On the other hand, EBRT plus ADT vs EBRT alone is associated with 10-year clinical disease-free survival improvement of, respectively, $47,7 \%$ vs $22,7 \%$, HR 0.42 (95\% CI 0.33-0.55; $\mathrm{p}<0.0001$, with no significant difference in major adverse events (cardiovascular mortality) between groups (Bolla et al., 2010).

However, until now, there is no high evidence evaluating the efficacy and safety of trimodal treatment $\mathrm{RP}+\mathrm{EBRT}+\mathrm{ADT}$ in patients with high-risk prostate cancer, which represents a subset disease with aggressive behavior, with higher recurrence, metastasis and cancer mortality rate (Chang et al., 2014). Because of the behavior of high-risk group of prostate cancer, we believe that combining the trimodal therapy can be more effective than combining two modalities. So, we are designing a randomized controlled trial to answer this question.

\section{METHODS}

\section{Trial design and study setting}

This trial is designed as a phase II, prospective, randomized, open- label, and multicenter trial. It will take place in five tertiary hospitals in Brazil that should count with a department of urology, clinical oncology and radiation oncology. All patients who fulfill eligibility criteria and provide their informed consent for participation will be able to participate in the study.

\section{Study Outcomes}

The primary endpoint is time to PSA recurrence, defined as time from the end of treatment to biochemical failure in 5 years. The definition of biochemical failure in both arms of treatment is considered as any PSA increase more than $2 \mathrm{ng} / \mathrm{mL}$ plus the PSA nadir value, regardless of the serum concentration of the nadir. PSA nadir is the lowest value reached after radiotherapy (Mottet et al., 2017).

Secondary end-points include metastasis free survival, defined as time to first clinical or radiological progression or death from any cause; overall survival, defined as the time from treatment of prostate cancer until death by any cause; adverse events according to graduation of Common Terminology Criteria for Adverse Events (CTCAE) v5.0 (Atkinson et al., 2016) and, quality of life assessed by Expanded Prostate Cancer Index Composite (EPIC) questionnaire (Wei, Dunn, Litwin, Sandler, \& Sanda, 2000).

\section{Eligibility criteria}

Inclusion Criteria:

- $\quad$ Male patient, from 18 years old to 75.

- Patients with histopathological confirmed prostate adenocarcinoma.

- High-risk localized prostate adenocarcinoma defined by the following criteria: PSA > 20 $\mathrm{ng} / \mathrm{mL}$, or Gleason score 8-10, or clinical stage T2c or higher.

- Patients signed the informed consent of the study.

- $\quad$ ECOG performance status 0-1.

- Charlson comorbidity index $\leq 3$ (Daskivich et al., 2011, 2013).

- Adequate organ function as per laboratory: Serum $\mathrm{Cr}<1.5 \mathrm{mg} / \mathrm{dl}$. Liver function tests ALT/AST - $<2$ times of normal value, Total Bilirubin $<2.5 \mathrm{mg} / \mathrm{dl}$, normal prothrombin time (PT) and activated Partial Thromboplastin Time (aPTT). CBC: Platelets $>100,000$, absolute neutrophil count (ANC) $>1500$, Hemoglobin $>10$ $\mathrm{mg} / \mathrm{dl}$.

- Agree to be enrolled in the study and willing to comply for investigation and receiving treatment as per study protocol. 


\section{Exclusion Criteria:}

- Prior treatment with chemotherapy, radiotherapy, hormonal therapy, surgery or immunotherapy for prostate cancer or other cancers.

- Metastatic disease evident by images like PET-CT scan, MRI images or bone scan.

- Evidence of organ dysfunction evident by laboratory tests above the cut value in inclusion criteria.

- Pathological lymph node positive disease after radical prostatectomy.

- Psychiatric condition that avoids complying to investigation or treatment according to study protocol

\section{Recruitment}

The health professionals who make the diagnosis of prostate cancer are usually urologists. We will target urological departments of all hospitals, urological offices and urological societies, and recruit urologists to refer their patients to this protocol, communicating the trial by emails, social media, and urological societies web pages.

Although clinical oncologists and radiation oncologists are usually not the professionals who make the initial diagnosis, they are also important partners of recruitment. Therefore, these specialties will also be included in our recruitment, using the same strategies mentioned above.

All the medium available for recruitment will be employed, in order to achieve the target population for the study, according to the design. The most important part of recruitment is the contact with health professional related to prostate cancer, as it has been described previously.

\section{Randomization and allocation concealment}

Participants will be randomly assigned to either RP+ EBRT + ADT group or EBRT + ADT group with a 1:1 allocation as per a computer generation randomization schedule stratified by site using permuted blocks of random sizes. The block sizes will not be disclosed, to ensure concealment.

\section{Interventions}

Patients will be randomized to either the trimodal treatment arm (intervention group- RP plus adjuvant EBRT plus ADT) or the bimodal treatment (comparator group - EBRT plus ADT).

\section{Intervention group}

The surgery will be an open radical retropubic prostatectomy that will remove prostate, capsule and seminal vesicles, and vesico-urethral anastomosis. Only one surgeon by site will performed the RP. Pelvic lymph node dissection will also be part of the procedure (Mottet et al., 2017). External Radiotherapy, in the intervention group will be administered until 16 weeks after the radical prostatectomy (Duthie \& Murphy, 2013; Mottet et al., 2017) in order to account as adjuvant radiation therapy. Patients will be submitted to adjuvant EBRT, with Intensity Modulated Radiotherapy (IMRT) technique, 66 Gy divided in 33 fractions (2 Gy daily dose).

The androgen deprivation therapy (ADT) will start on the first day of pelvic irradiation (Bolla et al., 2010), with Bicalutamide $50 \mathrm{mg} \mathrm{PO/day} \mathrm{starting} 15$ days before Goserelin, until 1 month after first do se of Goserelin. Goserelin $10.8 \mathrm{mg}$ SC every 3 months will be administered for 2 years (Zapatero, 2015).

\section{Comparator group}

The EBRT with IMRT technique, with total dose of 78 Gy divided in 39 fractions ( 2 Gy daily dose), in prostate and seminal vesicles (Kuban et al., 2011; Peeters et al., 2006). ADT will start on the first day of EBRT (Duthie \& Murphy, 2013), continue during EBRT, then for 2 years as adjuvant therapy. It will be used: Bicalutamide $50 \mathrm{mg} \mathrm{PO} /$ day starting 15 days before Goserelin, until 1 month after first dose of Goserelin. Goserelin 10.8 mg SC every 3 months will be administered for 2 years (Zapatero, 2015).

\section{Study Timeline}

The time frame for this trial will begin on the initial consent through the subject termination from the study. Each site will be responsible for screening and enrolling subjects per protocol. During the screening process, laboratory assessments will be completed to establish a baseline measure of the subject's condition prior to intervention.

Subjects will be randomized into two groups:

- Intervention group-Radical Prostatectomy + External Beam Radiation Therapy + Androgen Deprivation Therapy

- Comparator group - ADT + EBRT

Evaluations will be completed based on the specific arm the patient is randomized (See diagram below).

This study will perform the follow-up evaluations every 3 month ( \pm 2 weeks), and will include clinical evaluation, laboratory blood tests to assess PSA levels and evaluation of the adverse events in addition to assessment of quality of life by Expanded Prostate Cancer Index Composite (EPIC) questionnaire (Figure 1). 


\section{Timeline}

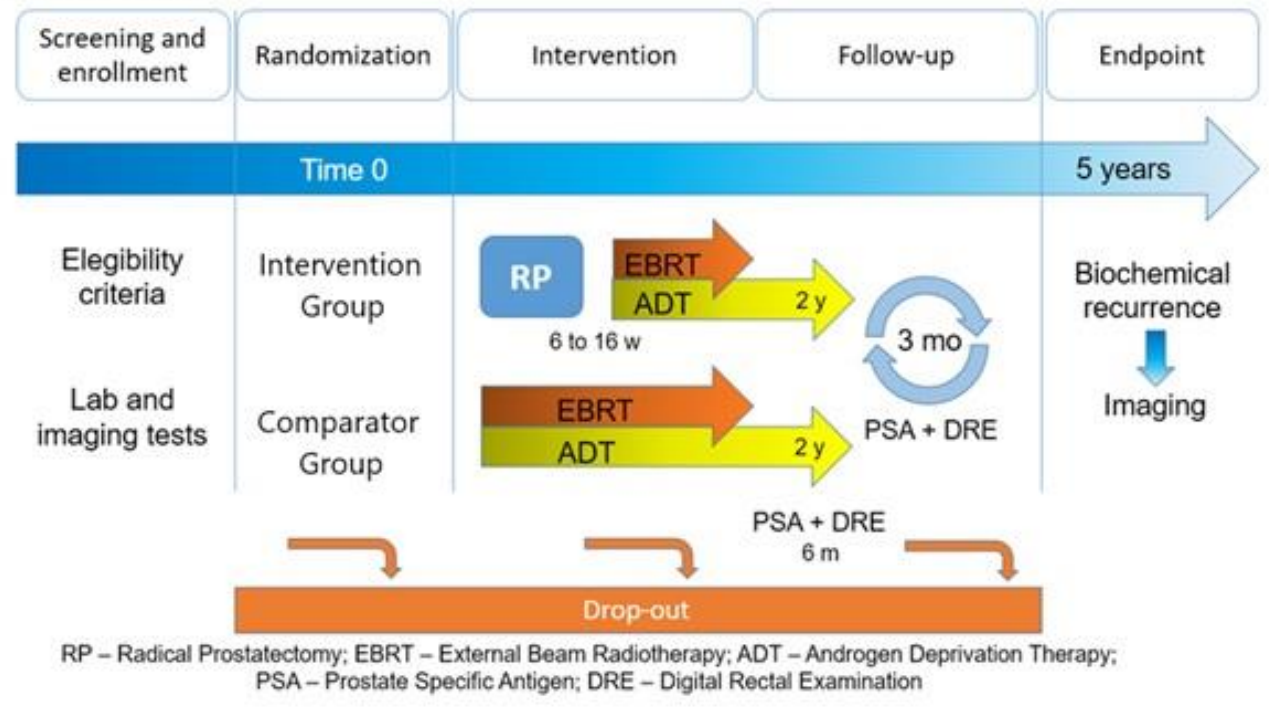

Figure 1. Timeline graphic of study design.

Subjects will be terminated from the study on year five. All adverse events will be followed up until they are resolved.

\section{Adherence}

We will adopt the following strategies:

- Detailed information strategy: In the recruitment phase we will provide detailed information by giving a comprehensive detail about the trial and make sure that the participant has a full achieved understanding of the study protocol.

- Social strategy: the protocol will involve family members, healthcare to promote study participation and social support for the participant.

- Health-care strategy: Healthcare providers who are involved in the study will be oriented about the study. They will attend awareness sessions about the importance of the relationship between the healthcare provider and patients in achieving a good level of adherence.

- Protocol strategies: Minimum effective frequency of medication/radiation should be selected and, minimum required follow-up visits should be scheduled.

- Logistical support: Transportation or free parking will be provided for the participants during the study visits. Schedule of the visits will be sent to the participant through their preferred contact method, a reminder (call or message) will be sent to the participant before the time of the medication dose, the surgery and radiotherapy session.

- Staff strategies: All involved research personnel will have sessions about adherence-enhancing behavior.

- Adherence monitoring: Diaries (paper or electronic according to patient preference) will be used for documenting the time of receiving pills \& any side effects or adverse effect happened. During any hospital admission the diary will be filled by the assigned nurse.

\section{Sample size calculation}

The sample size was calculated on the basis of the biochemical progression free survival measured by serum PSA level showed a hazard radio (HR) $=0.71$ (95\% CI $0.54,0.94 ; p=0.016$ ) in patients receiving radical prostatectomy with adjuvant radiotherapy (EBRT) (Thompson et al., 2009).

The following parameters to calculate the sample size were considered: $d=5 \%$, power $80 \%$, and withdrawn percentage $=15 \%$, percentage that has been added in the calculation. The number of patients required per group is 161 , resulting in a total of 322 subjects for the study. The program used was Stata/IC 15.1 (Stata Corp, TX, USA).

\section{Statistical Analysis for primary and secondary outcomes}

Statistical analysis for primary and secondary outcomes will consider both intention-to-treat (ITT) and perprotocol analysis (PP). Baseline characteristics such as 
age, BMI, smoking status, comorbidities, medications and PSA will be compared between groups. Continuous variables will be evaluated for normality using either histograms and will be expressed as mean +/- SD. Otherwise data will be expressed as median [interquartile ranges] and compared with Mann-Whitney test for nonparametric variable.

The primary outcome will be time to PSA recurrence after 5 year of follow-up. This will be plotted using Kaplan-Meier curves for group intervention $(\mathrm{RP}+\mathrm{EBRT}+\mathrm{ADT})$ versus group comparator (EBRT+ADT). The curves for both groups will be compared using log-rank test. Cox regression model will be used to adjust for covariates such as baseline PSA, age, Gleason Score, Tumor stage, smoking status and BMI.

For the secondary outcomes, metastasis free survival and overall survival will be analyzed the same way as the main outcome, and adverse events (such as urinary incontinence, and erectile dysfunction) rates at 5 years will be compared using chi-squared or Fisher's exact test for categorical variables. Quality of life using EPIC score will be compared between groups using Student t-test (or Mann-Whitney if not normally distributed). Statistical significance will be considered at two-sided alpha level of 0.05 .

The adjustment by site will be analysed by logistic regression. Statistical analysis will be performed using Stata/IC 15.1 (StataCorp, TX, USA).

\section{DISCUSSION}

Localized high-risk prostate cancer patients have two important features: oncologists are in front of a very aggressive disease, which may confer a recurrence rate of $50 \%$ in ten years after definitive treatment (Mottet et al., 2017) and, disease is confined to the prostate gland, which means that treatment in the beginning has a curative intent.

The standard treatment for high-risk prostate cancer is combined (more than two kinds) and active (performing an action to directly destroy or remove the tumor) treatment. The Protect Trial (Hamdy et al., 2016), which randomized 1643 patients in a controlled trial to either receive active surveillance $(n=545)$, surgery $(n=553)$ or radiation therapy $(n=545)$ concluded that it is better to treat patients in an active fashion than performing active surveillance, in terms of progression free survival.

American (Mohler et al., 2019) and European (Mottet et al., 2017) guidelines encourage the use of multimodal treatment, and the main alternatives describe treatment with radiation therapy and androgen deprivation therapy, or the inclusion of brachytherapy to this regime. Surgery is most of the time not popular among physicians in this particular set of patients for two particular reasons; the high chance of positive surgical margins and radical prostatectomy is considered a challenging operation that must be performed in high volume centers (Jang et al., 2018).

The reasoning behind our intervention is supported by the proof that combining treatments reduces the chance of local recurrence, which is high in this set of patients. A retrospective cohort study by Jang et al. (Jang et al., 2018), compared two bimodal treatments; radical prostatectomy (RP) and adjuvant radiation therapy (XRT) versus radiation therapy (XRT) and androgen deprivation therapy (ADT) for treating locally advanced and regionally advanced prostate cancer in 13856 patients. Patients with T3aNOM0, T3bNOM0, T4N0M0 disease who received RP+XRT versus XRT+ADT were less likely to die from prostate cancer [(HR, 4.22, 95\% CI 2.83-6.28), (HR, 1.84, 95\% CI 1.34-2.53), and (HR, 2.31, 95\% CI 1.05-5.12), respectively] and less likely to die from any cause [(HR, 1.75, 95\% CI 1.45-2.11), (HR, 1.49, 95\% CI 1.22-1.84), and (HR, 1.62, 95\% CI 1.00-2.64), respectively]. As a conclusion, adjusted 10-year prostate cancer - specific survival and 10-year overall survival favored men who underwent RP and XRT when compared to XRT and ADT. In our knowledge, there are not any randomized controlled trials related to this kind of approach, and in light of the lack of evidence, we think our protocol will contribute with an important piece of information related to this matter.

\section{CONCLUSIONS}

Given the fact that there are not any randomized controlled trials linked to the trimodal treatment that we are proposing, and after evaluating results from observational and retrospective studies, we think that the multimodal treatment we are trying to proof safe and effective, is needed in the field of prostate cancer and its results may contribute to change standard treatment in this set of patients by reducing significantly local recurrence and offering a potential cure for this malady.

\section{Author Affiliations}

1 Dirección Científica y Académica, Oncosalud AUNA, Lima, Perú. 2 Instituto Brasileiro de Controle de Câncer (IBCC Oncologia).

3 Urology department, AlWakra Hospital, Hamad Medical Corporation, Doha-Qatar.

4 West Hertfordshire NHS Trust, UK.

5 Respiratory Medicine Department, University Hospital Bern, Inselspital, Bern-Switzerland.

6 Trauma and Emergency Surgery Intensive Care Unit - Hospital das Clínicas da Universidade de São Paulo - HCFMUSP - Brazil.

7 Medical School Coordinator. Universidade Estadual do Oeste do Paraná. Campus Cascavel/PR/Brazil. 
8 Qatar Organ Donation Center - Hiba,HMC Doha Qatar.

9 State University of Londrina (UEL) - Brazil.

10 Assistant doctor of Otolaryngology Department of Santa Casa de Misericórdia de São Paulo, Brazil.

11 Hidromed, Guatemala.

12 Centro Universitário Lusíada (UNILUS) - Brazil.

13 Servicio Nacional De Salud, Universidad Católica Del Cibao, República Dominicana.

14 GI Endoscopy Unit, Department of Gastroenterology, Hospital das Clinicas of the University of São Paulo Medical School, Sao Paulo, Brazil.

15 Alumnus PPCR Class 2019.

16 Escuela de Medicina del Tecnologico de Monterrey, Mexico. 17 Surgical Intensive Care Unit, Anesthesiology Division, Hospital das Clínicas, University of São Paulo Medical School. HCFMUSP.

18 Alumnus PPCR Class 2019.

19 Centro de Estudios Clinicos ICIM, Universidad del Desarrollo, Chile.

20 Neurosurgery Department, Uopeccan Cancer Hospital, Cascavel, Brazil.

21 Physical Medicine and Rehabilitation. University Hospital Albacete. Spain.

22 Department of General, Visceral and Vascular Surgery, University Hospital Jena, Jena, Germany.

23 Faculdade de Medicina do ABC, Brazil.

24 Researcher Laboratório of Microsurgery and Plastic Surgery, Universidade de São Paulo, Brazil.

\section{REFERENCES}

Atkinson, T. M., Ryan, S. J., Bennett, A. V., Stover, A. M., Saracino, R. M., Rogak, L. J., ... Basch, E. (2016, August 1). The association between clinicianbased common terminology criteria for adverse events (CTCAE) and patient-reported outcomes (PRO): a systematic review. Supportive Care in Cancer, Vol. 24, pp. 3669-3676. https://doi.org/10.1007/s00520-0163297-9

Bolla, M., Van Poppel, H., Tombal, B., Vekemans, K., Da Pozzo, L., De Reijke, T. M., ... Collette, L. (2012). Postoperative radiotherapy after radical prostatectomy for high-risk prostate cancer: Long-term results of a randomised controlled trial (EORTC trial 22911). The Lancet, 380(9858), 2018-2027. https://doi.org/10.1016/S0140-6736(12)61253-7

Bolla, M., Van Tienhoven, G., Warde, P., Dubois, J. B., Mirimanoff, R. O., Storme, G., ... Collette, L. (2010). External irradiation with or without long-term androgen suppression for prostate cancer with high metastatic risk: 10-year results of an EORTC randomised study. The Lancet Oncology, 11(11), 1066-1073. https://doi.org/10.1016/S14702045(10)70223-0

Chang, A. J., Autio, K. A., Roach, M., \& Scher, H. I. (2014). High-risk prostate cancer-Classification and therapy. Nature Reviews Clinical Oncology, Vol. 11, pp. 308-323. https://doi.org/10.1038/nrclinonc.2014.68

Daskivich, T. J., Chamie, K., Kwan, L., Labo, J., Dash, A., Greenfield, S., \& Litwin, M. S. (2011). Comorbidity and competing risks for mortality in men with prostate cancer. Cancer, 117(20), 4642-4650. https://doi.org/10.1002/cncr.26104

Daskivich, T. J., Fan, K. H., Koyama, T., Albertsen, P. C., Goodman, M., Hamilton, A. S., ... Penson, D. F. (2013). Effect of age, tumor risk, and comorbidity on competing risks for survival in a U.S. population-based cohort of men with prostate cancer. Annals of Internal Medicine, 158(10), 709-717. https://doi.org/10.7326/0003-4819-158-10-201305210-00005

Duthie, J. B., \& Murphy, D. G. (2013, June). Re: Postoperative radiotherapy after radical prostatectomy for high-risk prostate cancer: Long-term results of a randomised controlled trial (EORTC Trial 22911). European Urology, Vol. 63, pp. 1131-1132. https://doi.org/10.1016/j.eururo.2013.03.021
Hamdy, F. C., Donovan, J. L., Lane, J. A., Mason, M., Metcalfe, C., Holding, P., ... Neal, D. E. (2016). 10-Year Outcomes after Monitoring, Surgery, or Radiotherapy for Localized Prostate Cancer. New England Journal of Medicine, 375(15), 1415-1424. https://doi.org/10.1056/NEJMoa1606220

Jang, T. L., Patel, N., Faiena, I., Radadia, K. D., Moore, D. F., Elsamra, S. E., ... LuYao, G. L. (2018). Comparative effectiveness of radical prostatectomy with adjuvant radiotherapy versus radiotherapy plus androgen deprivation therapy for men with advanced prostate cancer. Cancer, 124(20), 4010-4022. https://doi.org/10.1002/cncr.31726

Kuban, D. A., Levy, L. B., Cheung, M. R., Lee, A. K., Choi, S., Frank, S., \& Pollack, A. (2011). Long-term failure patterns and survival in a randomized doseescalation trial for prostate cancer. Who dies of disease? International Journal of Radiation Oncology Biology Physics, 79(5), 1310-1317. https://doi.org/10.1016/j.jirobp.2010.01.006

Mohler, J. L., Antonarakis, E. S., Armstrong, A. J., D’Amico, A. V., Davis, B. J., Dorff, T., ... Freedman-Cass, D. A. (2019). Prostate cancer, version 2.2019. JNCCN Journal of the National Comprehensive Cancer Network, 17(5), 479-505. https://doi.org/10.6004/jnccn.2019.0023

Mottet, N., Bellmunt, J., Bolla, M., Briers, E., Cumberbatch, M. G., De Santis, M., ... Cornford, P. (2017). EAU-ESTRO-SIOG Guidelines on Prostate Cancer. Part 1: Screening, Diagnosis, and Local Treatment with Curative Intent. European Urology, 71(4), 618-629. https://doi.org/10.1016/j.eururo.2016.08.003

Peeters, S. T. H., Heemsbergen, W. D., Koper, P. C. M., Van Putten, W. L. J., Slot, A., Dielwart, M. F. H., ... Lebesque, J. V. (2006). Dose-response in radiotherapy for localized prostate cancer: Results of the Dutch multicenter randomized phase III trial comparing $68 \mathrm{~Gy}$ of radiotherapy with 78 Gy. Journal of Clinical Oncology, 24(13), 1990-1996. https://doi.org/10.1200/JCO.2005.05.2530

Siegel, R. L., Miller, K. D., \& Jemal, A. (2019). Cancer statistics, 2019. CA: A Cancer Journal for Clinicians, 69(1), 7-34. https://doi.org/10.3322/caac.21551

Thompson, I. M., Tangen, C. M., Paradelo, J., Lucia, M. S., Miller, G., Troyer, D., ... Crawford, E. D. (2009). Adjuvant Radiotherapy for Pathological T3NOMO Prostate Cancer Significantly Reduces Risk of Metastases and Improves Survival: Long-Term Followup of a Randomized Clinical Trial. Journal of Urology, 181(3), 956-962. https://doi.org/10.1016/j.juro.2008.11.032

Wei, J. T., Dunn, R. L., Litwin, M. S., Sandler, H. M., \& Sanda, M. G. (2000). Development and validation of the Expanded Prostate Cancer Index Composite (EPIC) for comprehensive assessment of health-related quality of life in men with prostate cancer. Urology, 56(6), 899-905. https://doi.org/10.1016/\$0090-4295(00)00858-X

Zapatero, A. (2015). High-dose radiotherapy with short-term or long-term androgen deprivation in localised prostate cancer (DART01/05 GICOR): A randomised, controlled, phase 3 trial. The Lancet Oncology, 16(3), 320-327. https://doi.org/10.1016/S1470-2045(15)70045-8 